OPEN ACCESS

Edited by:

Michal Mego,

Campus Bio-Medico University, Italy

Reviewed by:

Elena Ranieri,

University of Foggia, Italy

Rasha Abu Eid,

University of Aberdeen,

United Kingdom

${ }^{*}$ Correspondence:

Giuseppe Procopio

giuseppe.procopio@

istitutotumori.mi.it

Specialty section:

This article was submitted to

Genitourinary Oncology,

a section of the journal

Frontiers in Oncology

Received: 31 May 2020

Accepted: 27 July 2020

Published: 12 August 2020

Citation:

Raimondi A, Sepe P, Zattarin E, Mennitto A, Stellato $M$, Claps $M$,

Guadalupi V, Verzoni E, de Braud F and Procopio G (2020) Predictive Biomarkers of Response

to Immunotherapy in Metastatic Renal

Cell Cancer. Front. Oncol. 10:1644.

doi: 10.3389/fonc.2020.01644

\section{Predictive Biomarkers of Response to Immunotherapy in Metastatic Renal Cell Cancer}

\author{
Alessandra Raimondi', Pierangela Sepe ${ }^{1}$, Emma Zattarin ${ }^{1}$, Alessia Mennitto', \\ Marco Stellato ${ }^{1}$, Melanie Claps ${ }^{1}$, Valentina Guadalupi ${ }^{1}$, Elena Verzoni', \\ Filippo de Braud ${ }^{1,2}$ and Giuseppe Procopio ${ }^{1 *}$ \\ ' Department of Medical Oncology, Fondazione IRCCS Istituto Nazionale dei Tumori, Milan, Italy, ${ }^{2}$ Oncology \\ and Hemato-Oncology Department, University of Milan, Milan, Italy
}

Introduction: In the last decades, the therapeutic decision-making approach to metastatic renal cell cancer ( $\mathrm{mRCC}$ ) has dramatically changed thanks to the introduction in the treatment scenario of, first, anti-angiogenic agents and, afterward, immunecheckpoint inhibitors (ICls). Immunotherapy is now the standard of care in pretreated $m R C C$ patients and has recently entered even the first line setting. Nevertheless, in $\mathrm{mRCC}$ as well as in other tumor settings, a durable and clinically meaningful benefit from treatment with $\mathrm{ICls}$ is not obtained for all patients treated. Therefore, the necessity to identify and validate predictive biomarkers of response to immunotherapy has emerged, in order to design the optimal treatment strategy for mRCC patients.

Discussion: In this review, we present and discuss the most promising predictive biomarkers of response to $\mathrm{ICls}$ in $\mathrm{mRCC}$ with the recent data available. In details, the first marker that was investigated is the immunohistochemical expression of programmed death receptor ligand 1 (PD-L1), showing a negative prognostic role in mRCC, but the debate about its potential predictive value is still open. Additionally, the high heterogeneity in PD-L1 determination methods adds complexity to this issue. Second, the tumor mutational or neoantigen burden is an emerging biomarker of increased response to immunotherapy, hypothesizing that the higher the TMB, the higher is the production of neoantigens, and thus the stimulation of anti-tumor immune response, even though controversial results have been obtained. Third, the tumor microenvironment, namely the different populations of the immune infiltrate, plays a key role in tumor progression and in the response to immunotherapy. Finally, several studies have collected evidence on the potential association of the occurrence of immunerelated adverse events (irAEs) with the benefit from ICls, first in non-small cell lung cancer (NSCLC) and melanoma, and recently even in mRCC.

Conclusion: Several promising biomarkers of response to immunotherapy with ICls have been identified, though without conclusive results upon their potential predictive value in mRCC. Therefore, the results of the exploratory analyses of the recently presented first-line trials and hopefully of future prospective, biomarker-driven studies could provide useful tools to be applied in the everyday clinical practice.

\footnotetext{
Keywords: biomarker, immunotherapy, metastases, PD-L1, renal cell carcinoma, tumor mutational burden
} 


\section{INTRODUCTION}

Renal cell cancer (RCC) represents about $3 \%$ of all solid tumors worldwide, with an estimated incidence of approximately 330,000 new cases per year (1). RCC is diagnosed at an advanced stage in around one third of cases, and metastases develop in another $30 \%$ of patients after initial nephrectomy for localized disease (2). The prognosis of metastatic RCC (mRCC) is generally considered poor, with a predicted 5 -year survival rate lower than $20 \%$, even though the patients' outcome could be stratified according to several prognostic factors (3).

In the past decades, thanks to a deeper understanding of the biological and molecular disease scenario, the clinical approach to mRCC has undergone a dramatic change with the introduction of novel drugs (4). First, the anti-angiogenic therapies targeting the Vascular Endothelial Growth Factor (VEGF) and its receptor, and, subsequently, immunotherapy revolutionized the armamentarium for the treatment of mRCC. Therefore, the refined therapeutic decision-making process allowed to reach a remarkable improvement in patients' outcome in terms of progression-free survival (PFS) and overall survival (OS), as well as in patients' quality of life $(5,6)$.

In details, the targeting of the immune system deeply changed the disease course and the prognosis of patients affected by several tumors, among which non-small cell lung cancer (NSCLC), melanoma and RCC. The introduction of the immune-checkpoint inhibitors (ICIs) targeting first the Cytotoxic T-Lymphocyte Antigen 4 (CTLA4) and subsequently the programmed death receptor 1 (PD1) and its ligand (PD-L1) provided a new therapeutic standard and a changing paradigm in different tumor settings (7).

For what concerns mRCC, immunotherapy entered the clinical scenario in patients who had received a prior line with anti-angiogenic agents, in light of the results of the CheckMate 025 trial $(8,9)$. The study compared the anti-PD1 agent nivolumab with the standard of care everolimus and showed a statistically significant and clinically meaningful improvement in terms of survival with a more favorable safety profile for immunotherapy, leading to the approval from Food and Drug Administration (FDA) and European Medical Association (EMA) $(10,11)$.

Subsequently, in order to optimize the potential benefit of immunotherapy, ICIs have been investigated in the firstline setting, either alone (i.e., nivolumab plus ipilimumab) or associated with anti-vascular agents (i.e., avelumab plus axitinib, pembrolizumab plus axitinib and atezolizumab plus bevacizumab), thanks to several clinical trials that recently presented their first results (12). Altogether, the available data showed an overall improvement of the treatment outcomes with immunotherapy-based combinations over the anti-vascular tyrosine kinase inhibitor (TKI) monotherapy. In details, in the CheckMate 214 trial, a significant benefit in OS and overall response rate (ORR) for nivolumab plus the anti-CTLA4 agent ipilimumab over sunitinib was reported for intermediate/poor risk patients (13), and the Javelin Renal 101 study showed a 6.6-month increase in PFS for the anti-PD-L1 agent avelumab combined with axitinib as compared to sunitinib monotherapy
(14). Moreover, in the IMmotion151 trial, the combination of the anti-PD-L1 atezolizumab plus bevacizumab prolonged PFS as compared to sunitinib in patients with PD-L1 positive mRCC (15), and the KEYNOTE-426 study demonstrated a significant benefit in terms of OS, PFS and ORR for the anti-PD1 agent pembrolizumab plus axitinib versus sunitinib (16). In light of these results, the treatment paradigm for mRCC patients is undergoing a deep change, in particular in the first-line setting, since FDA and EMA recently approved the combination of nivolumab plus ipilimumab for intermediate and poor risk patients and the association of pembrolizumab plus axitinib independently from the risk category $(17,18)$.

Additionally, studies are underway to investigate whether a rechallenge with ICIs in later lines could be safe and effective. Initial evidence has been collected on this topic, suggesting that this strategy might be feasible, even though prospective ad hoc studies should be designed and conducted in order to potentially validate this therapeutic approach in mRCC patients (19).

Nevertheless, the benefit of immunotherapy is confined to a limited proportion of mRCC patients who achieve a significant and durable disease control from treatment with ICIs. Therefore, the identification of predictive biomarkers for response to immunotherapeutic agents represents an unmet clinical need in this disease setting, with the aim to improve the outcome of mRCC patients.

In this review, we summarize the most promising predictive biomarkers for response to immunotherapy and the available evidence collected on this topic in the setting of mRCC, as illustrated in Figure 1.

\section{BIOMARKERS}

\section{PD-L1}

The first and most studied potential biomarker for response to anti-PD1/PD-L1 agents is the expression of PD-L1. The clinical significance of the expression of PD-L1 on tumor cells and/or on the immune cells infiltrating the tumor assessed with immunohistochemistry (IHC) was initially identified in the first clinical study investigating the anti-PD1 agent nivolumab and, afterward, it has been widely studied in several settings (20).

The reported rate of PD-L1 expression in the different tumors is endowed with a high variability across studies, and, considering specifically the neoplastic diseases with enhanced response to ICIs, namely NSCLC, melanoma and RCC, it ranges between 14 and $100 \%$ (21). Nevertheless, even neoplasms with reduced sensitivity to immunotherapy, for example colorectal cancer or sarcoma, show a comparable IHC expression of PD-L1, thus underlining the limitations of this biomarker and giving an insight on the complexity of the scenario (22).

Considering the association of the expression of PD-L1 with prognosis, several studies collected evidence on a potential negative prognostic value of PD-L1 overexpression in gastric, hepatocellular, esophageal, pancreatic, ovarian, and bladder cancer, whereas a positive value in breast cancer and merkel cell carcinoma and controversial results in lung cancer, colorectal cancer, and melanoma (23). For example, in advanced/metastatic 


\section{BIOMARKERS}
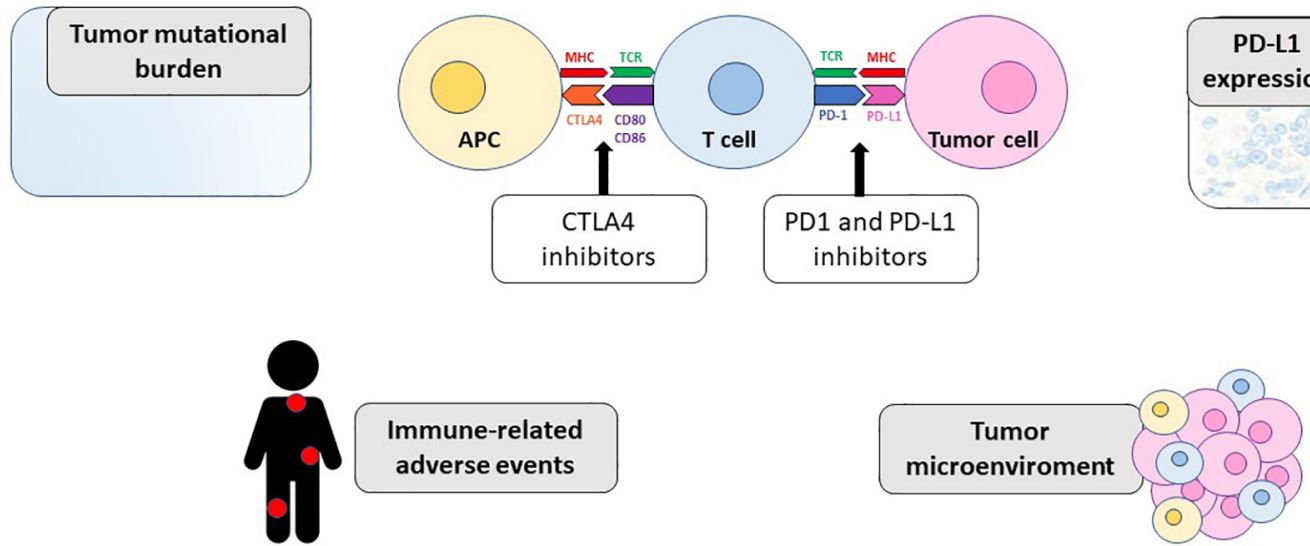

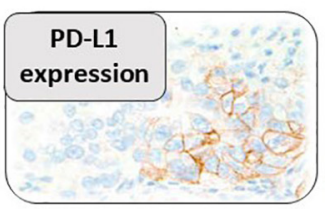

FIGURE 1 | The potential biomarkers of response to immunotherapy discussed in the review are illustrated, together with the mechanism of action of immune-checkpoint inhibitors. CTLA4, Cytotoxic T-Lymphocyte Antigen 4; PD-1, programmed death receptor 1; PD-L1, programmed death receptor ligand 1; MHC, major histocompatibility complex; TCR, T cell receptor; APC, antigen presenting cell; CD, cluster of differentiation.

NSCLC, a systematic literature review did not observe an association between PD-L1 expression and patients or tumor characteristics but, in the majority of studies, a high expression of PD-L1 correlated with reduced survival (24). Nevertheless, the global level of evidence does not allow to drive definitive conclusions on this topic.

In the setting of mRCC, the negative prognostic role of the expression of PD-L1 on tumor cells has been reported in several studies $(25,26)$. Renal cancers showing the expression of PD-L1 on tumor cells display a higher tumor stage, a worse response to TKI therapy and a poorer prognosis (27). In details, in two post hoc analyses of the COMPARZ study and the METEOR and CABOSUN trials, respectively, PD-L1 expression on tumor cells was associated with shorter survival in mRCC patients, irrespectively of the type of targeted therapy received $(28,29)$. Furthermore, in a metanalysis including more than 1300 patients, the expression of PD-L1 in RCC seemed to confer a poorer outcome, approximately doubling the risk of death, and this finding was confirmed after the restriction of the analysis to patients affected by clear cell histology RCC, in case of advanced disease and in cases in which PD-L1 was evaluated uniquely with IHC (30).

On the other hand, dealing with the predictive value of PDL1 expression for an enhanced response to immunotherapy with PD1/PD-L1 inhibitors, the global burden of evidence collected in several tumors showed that a higher expression of PD-L1 may be able to predict the responsiveness to ICIs, even though in most cancers the relevance of this correlation is insufficient to validate $\mathrm{PD}-\mathrm{L} 1$ as a predictive biomarker potentially applicable in the clinical setting $(31,32)$. In melanoma, in trials investigating nivolumab and pembrolizumab in the advanced/metastatic setting, patients whose tumors displayed the positivity for PD-L1 had a higher response rate and a prolonged survival outcome, however, robust and durable responses to treatment were observed even in a considerable proportion of $\mathrm{PD}-\mathrm{L} 1$ negative patients $(33,34)$. Therefore, $\mathrm{PD}$ L1 expression is not currently used in the clinical practice as a biomarker to guide patients' selection for treatment. On the other hand, in advanced NSCLC, the results of the recent studies evidenced how the IHC expression of PD-L1 and its cutoff are fundamental in the therapeutic decision-making of the non-oncogene-addicted disease, whether choosing the anti-PD1 monotherapy, chemotherapy or the combination of chemotherapy and immunotherapy in first-line (35).

In $\mathrm{mRCC}$, the potential predictive value for response to immunotherapy of PD-L1 IHC expression is still controversial and the results obtained in the exploratory analyses of the landmark clinical trials investigating ICIs in this disease are inconclusive $(8,13-16)$. In details, in the CheckMate 025 trial, that proved the superiority of nivolumab over the standard of care everolimus in pre-treated patients, the expression of $\mathrm{PD}$ L1 was associated with poor survival independently from the treatment received, but not with response to nivolumab. In details, in patients with PD-L1 $\geq 1 \%$ assessed on tumor cells, an inferior OS was observed as compared to those with PD$\mathrm{L} 1<1 \%$ in both treatment arms, with a similar trend for the 5\% cutoff. On the other hand, nivolumab was superior to everolimus irrespectively of PD-L1 expression, since median OS resulted to be 21.8 versus 18.8 months and 27.4 versus 21.2 months in $\mathrm{PD}-\mathrm{L} 1 \geq 1$ and $<1 \%$ subgroups, respectively (8). Moving to the first-line setting, in the CheckMate 214 trial, a longer PFS was observed for the combination immunotherapy ipilimumab plus nivolumab over sunitinib in IMDC intermediate-poor risk patients for PD-L1 positive tumors, that is $1 \%$ or greater (median 
PFS 22.8 versus 5.9 months), but not for negative ones, that is less than $1 \%$ (median PFS 11 versus 10.4 months). Conversely, an improvement in ORR and OS for immunotherapy over the anti-vascular agent was reported independently from the tumor PD-L1 expression level, even though the magnitude of benefit was higher in patients with PD-L1 $\geq 1 \%$ (13). Recently the phase 3 IMmotion 151 trial reported that the combination of atezolizumab plus bevacizumab was superior to sunitinib monotherapy in terms of PFS in patients affected by PD-L1 positive mRCC, with a more favorable safety profile. The trial included patients independently from the PD-L1 status, that represented a stratification factor, and the magnitude of benefit seemed increased in patients with PD-L1 $\geq 1 \%$ as compared to the intention-to-treat trial population (15). These evidences are in line with the results of the phase 2 IMmotion 150 study, that was designed with two atezolizumab-based arms (monotherapy or in combination with bevacizumab) as compared to sunitinib, and evidenced a trend in a progressively increasing treatment efficacy with higher PD-L1 expression (defined as any intensity staining in immune cells covering absent $/<1 \%$ (IC0), $\geq 1 \%$ to $<5 \%$ (IC1), $\geq 5 \%$ to $<10 \%$ (IC2) or $\geq 10 \%$ (IC3) of tumor area assessed by SP142 assay) mainly in the atezolizumab/bevacizumab arm (36). Furthermore, in the JAVELIN Renal 101 trial, where PDL1 positivity was assessed on immune cells infiltrating the tumor with a cutoff of $1 \%$, the combination of avelumab plus axitinib showed a PFS and ORR advantage as compared to sunitinib monotherapy, and the benefit was substantially comparable in the overall population and in the PD-L1 positive subgroup (median PFS 13.8 versus 8.4 months and 13.8 versus 7.2 months; ORR $51.4 \%$ versus $25.7 \%$ and $55.2 \%$ versus $25.5 \%$, in avelumab plus axitinib versus sunitinib arms, respectively), even though with a non-significant trend toward improved PFS in the PD-L1 negative group $(14,37)$. Finally, in the KEYNOTE-426 trial, the combination of pembrolizumab plus axitinib showed a benefit over sunitinib in terms of PFS, OS and ORR, irrespectively of PD-L1 expression (assessed with the combined score, including tumor cells, lymphocytes and macrophages), although the hazard ratios for $\mathrm{PFS}$ and $\mathrm{OS}$ seemed to underline an enhanced advantage in $\mathrm{PD}-\mathrm{L} 1 \geq 1$ versus $<1$ subgroups [0.62 (95\%CI $0.47-$ $0.80)$ versus 0.87 (95\%CI $0.62-1.23)$ for PFS and 0.54 (95\%CI $0.35-0.84$ ) versus 0.59 (95\%CI 0.34-1.03) for OS] (16).

Overall, these results suggest that the expression of PDL1 in mRCC is not able to completely predict the potential responsiveness of tumor to anti PD1/PD-L1 ICIs, thus, the role of PD-L1 as a predictive therapeutic biomarker remains controversial and warrants further investigation in ad hoc designed studies.

The main limitations of PD-L1 expression are related both to the assessment method and to the tumor heterogeneity (22). The first weakness, connected with the technique used for the IHC analysis, is the elevated variability among the different determination methods available, since each PD1/PDL1 inhibitor is endowed with its own companion antibody (e.g., Dako, Leica platform, Ventana Medical System). In details, the scoring systems are not homogeneous for the target cells assessed, whether tumor cells, immune cells infiltrating the neoplastic stroma or the combination of both, and the positivity cutoff has not been validated (e.g., 1, 5, and 10\%) $(32,38)$. Additionally, it is not clear whether the expression of PD-L1 or PD1 should be assessed, and, additionally, the role of PD-L2, the other ligand of PD1, is still to be fully elucidated (39). Finally, the expression of PD-L1 should not be considered static but dynamic, since it may vary during the tumor natural history or as a consequence of antineoplastic treatments and, additionally, PD-L1 expression shows an elevated heterogeneity both intratumoral and between the primary tumor and the distant metastases, potentially explaining the high responses obtained in cases in which the primary tumor or one lesion had been characterized as PD-L1 negative (40).

\section{Tumor Mutational Burden/Neoantigen Burden}

The tumor mutational burden (TMB) is a recently defined emerging biomarker of increased response to immunotherapy. The definition of TMB is the total number of mutations per coding area of tumor genome, measured as mutations per megabase (mutations/Mb). The genomic alterations occurring in tumor cells display the capacity to generate tumor-specific antigens (neoantigens), potentially processed and presented on the surface of cancer cells and therefore recognized by the T cells, eliciting the anti-tumor response (41). The potential association of TMB with sensitivity to ICIs is based on the hypothesis that in tumors with high TMB there is an increased production of surface neoantigens, thus stimulating the anti-tumor immune system response (42). TMB has been investigated in several tumor settings, mainly NSCLC and melanoma, as a stratification marker to predict the response to immune agents, showing promising yet inconclusive results $(43,44)$. In details, in the CheckMate 227 trial, patients with NSCLC were randomized to receive different nivolumab-based regimens versus chemotherapy and the results showed that those displaying a TMB equal or higher than 10 mutations/Mb had an increased PFS with the treatment combining nivolumab and ipilimumab (43). The non-conclusive results obtained may be partially explained by the heterogeneity in the definition and assessment methods of TMB, as well as of positivity cutoffs, since TMB could be assessed by the estimation of somatic mutations with whole exome sequencing or by the direct sequencing of panels of cancer-related genes using next-generation sequencing. Moreover, these techniques are not widely available, limiting the potential application of this tool in the clinical practice (45).

In the setting of mRCC, initial evidence has been collected on this topic in recent studies. TMB scores display a deep variability through the different histological subtypes of RCC, ranging from a very low level in chromophobe type to a comparable value of TMB in clear cell and papillary tumors and, additionally, TMB is not associated with the clinically defined prognostic groups according to IMDC and MSKCC (46). Interestingly, despite its high rate of response to immunotherapy, RCC is endowed with an average low TMB, only 1.1 mutations/Mb, as compared with melanoma and NSCLC that display values ranging from 10 to 400 mutations/Mb (46, 47). Nevertheless, RCC represents the tumor endowed with the highest proportion of insertion/deletion alterations, 
that represent a highly immunogenic mutational class, thus potentially leading to the production of neo-antigens (48). The results regarding the prognostic value of $\mathrm{TMB}$ and its potential correlation with response to immunotherapy in the setting of mRCC are conflicting. Regarding the association with prognosis, the literature evidences are controversial, since some studies report a trend toward improved survival with the increase of $\mathrm{TMB}$, while others observed a negative prognostic value $(49,50)$. Concerning the predictive value of TMB, TMB and other biomarkers were evaluated in a subset of 35 pretreated mRCC patients enrolled in the phase $1 \mathrm{~b}$ study of nivolumab (NCT01358721), showing that only a specific and small proportion of tumors could benefit from treatment, and the results were then validated in another independent cohort of patients treated with nivolumab or atezolizumab (51). Moreover, in a retrospective dataset of $34 \mathrm{mRCC}$ patients, TMB did not correlate neither with survival nor with PD-L1 expression on tumor cells (52). Additionally, in the exploratory analysis of the IMmotion 150 trial, no association between TMB or neoantigen burden with an enhanced clinical benefit from nivolumab was shown (36).

Taken all these data into consideration, ad hoc prospective trials are awaited, with the aim to unravel the potential predictive role of TMB in the setting of mRCC. The ongoing NIVES study (NCT03469713), investigating the combination of nivolumab with stereotactic radiotherapy in pretreated mRCC patients, recently presented the first data, and its exploratory analyses could provide evidence on this topic in the next future (53).

\section{Microenvironment/Signatures}

In the complex scenario of cancer evolution and of response to immunotherapy, the tumor microenvironment plays a key role, since the different subpopulations of immune cells that infiltrate the tumor and their interplay may deeply influence the sensitivity to ICIs.

Renal cell cancer is endowed with a heterogeneous population of tumor infiltrating immune cells, that include $\mathrm{T}$ and $\mathrm{B}$ lymphocytes as well as innate immunity cells like monocytes and macrophages. A remarkable burden of evidence has been collected on the characterization of the tumor infiltrate in RCC, highlighting its impact on patients' clinical outcome and suggesting a potential role as a target in cancer treatment (54). However, conflicting data have been obtained as far as now. In details, some studies have shown that the infiltration of effector $\mathrm{T}$ cells, such as CD8+ lymphocytes, and M1 macrophages may confer a better prognosis, whereas regulator $\mathrm{T}$ cells, such as $\mathrm{T}$ regs, and M2 macrophages could be associated with a poorer outcome (55-58). Conversely, other trials have reported a poor prognosis in cases displaying a high intra- and peri-tumoral $\mathrm{T}$ CD8+ cells density, potentially related to the positive association between lymphocytes and tumor grade (59). Interestingly, it was reported that the expression of PD-L1 on tumor cells correlated with a higher infiltration of $\mathrm{T}$ CD8+ cells in the microenvironment of RCC, with potential implications for prognosis (54), that is influenced even by the presence and grade of activation of dendritic cells in the tumor, able to drive the anti-tumor immune reaction (60).
Concerning the potential predictive role of the immune infiltrate, in several tumors (such as melanoma, breast and colorectal cancer), it was reported that the presence of tumorinfiltrating lymphocytes is associated with an enhanced response to immunotherapy (61). Moreover, moving forward, specific genomic signatures, based on gene expression profiles predictive of immune system activation and anti-tumor response, have been developed and investigated in various cancer settings as potential biomarkers able to predict the response to immunotherapy (62). In patients with solid tumors treated with pembrolizumab in a clinical trial setting, higher $\mathrm{T}$ cell-inflamed gene expression profile scores were associated with increased response to treatment (63). In mRCC, a comprehensive analysis on patients enrolled in four clinical trials with nivolumab showed that the expression profiles involved in the regulation of metabolic and immune pathways correlated with the clinical outcome. In details, on the one hand, the overexpression of genes involved in metabolic functions (e.g., UGT1A) and, on the other, the increased expression of immune markers (e.g., BACH2 and CCL3) correlated with a poorer and higher response to treatment, respectively (64). Additionally, the exploratory analysis of the IMmotion150 trial reported that a T-effector immune gene signature displayed an association with the expression of PDL1 and the tumor infiltration of T CD8 + cells, resulting in an increased response to atezolizumab, with a higher ORR and a prolonged PFS (36). These data find their rationale in the potential immune-modulating reaction induced by the vascular inhibition via VEGF-blockade, able to promote the infiltration of $\mathrm{T}$ cells in the tumor microenvironment, thus potentiating the mechanism of action of ICIs (65). Further studies on this topic are awaited, given the complexity of the molecular and biological cancer scenario in mRCC.

\section{Immune-Related Adverse Events}

Another emerging topic in the setting of the identification of potential predictors of response to immunotherapy is the occurrence of immune-related adverse events (irAEs). In details, ICIs determine a peculiar spectrum of treatment-related toxicities, arising from an excessively augmented activity of the immune system and potentially involving any organ with a multifaceted variety of presentations $(66,67)$. The pathogenesis of irAEs may arise from the cross-reactivity between normal tissues and tumor neoantigens or from the alteration of the humoral and/or cell-mediated immunity, including immunosuppressive regulatory $\mathrm{T}$ cells and the production of antibodies by $\mathrm{B}$ cells. The most common ICIs-related toxicities include dermatological AEs, such as rash, vitiligo and pruritus, gastrointestinal, like diarrhea, colitis, hepatitis, amylase and lipase elevation, endocrine, such as thyroid disfunctions (hypothyroidism and/or hyperthyroidism) and hypophysitis, pulmonary, as pneumonitis, renal and systemic, like fever and fatigue (68). For what concerns specifically the renal function impairment, the main irAE is nephritis, either symptomatic or not, that consists in an inflammation of the kidney affecting the structure and limiting its functions. This is peculiarly relevant for RCC patients, since they may have a baseline reduction of renal function or a limited functional reserve. The guidelines for the management 
of immune-related nephritis are based upon the grading of the AEs and recommend to stop or even discontinue the treatment with ICIs, consult a nephrology specialist and, in selected cases, to start a corticosteroid treatment (67). A remarkable yet nonconclusive amount of data has been collected upon a possible correlation between the occurrence of irAEs and an increased benefit in terms of survival from ICIs, mainly in melanoma and NSCLC (69-71). Recently, even in the setting of mRCC, some studies have provided evidence of a consistent result. In details, in mRCC patients treated with nivolumab in the second and further line setting in a compassionate-use program, those reporting irAEs showed a significantly longer OS as compared to patients not experiencing immune-mediated toxicity (median OS not reached versus 16.8 months) (72). Comparable results have been obtained in a retrospective dataset of Asian patients with mRCC receiving nivolumab, highlighting how irAEs were associated with improved PFS and OS (73).

Nevertheless, the specific criteria for the definition of irAEs have not been established so far and whether any toxicity arising from an immune-mediated mechanism or specific classes of irAEs display a meaningful correlation with response to immunotherapy remains to be elucidated yet, thus warranting further investigation in this setting.

\section{CONCLUSION}

Immunotherapy revolutionized the therapeutic strategy and the clinical outcome of patients affected by several different tumors, among which RCC. Nevertheless, not all patients derive a significant and durable benefit from immunotherapy with ICI, indeed, this underlines the unmet clinical need of identifying reliable predictive markers of response to immune agents.

\section{REFERENCES}

1. Capitanio U, Montorsi F. Renal cancer. Lancet. (2016) 387:894-906. doi: 10. 1016/S0140-6736(15)00046-X

2. Ferlay J, Soerjomataram I, Ervik M, Dikshit R, Eser S, Mathers C, et al. GLOBOCAN 2012 v1.0, Cancer Incidence and Mortality Worldwide: IARC CancerBase No. 11 [Internet]. Lyon: International Agency for Research on Cancer (2013).

3. Heng DY, Xie W, Regan MM, Harchman LC, Bjarnason GA, Vaishampayan $\mathrm{UN}$, et al. External validation and comparison with other models of the international metastatic renal-cell carcinoma database consortium prognostic model: a population-based study. Lancet Oncol. (2013) 14:141-8. doi: 10.1016/ S1470-2045(12)70559-4

4. Ratta R, Verzoni E, Di Maio M, Grassi P, Colecchia M, Fucà G, et al. Exposure to multiple lines of treatment and survival of patients with metastatic renal cell carcinoma: a real-world analysis. Clin Genitourin Cancer. (2018) 16:e735-42. doi: 10.1016/j.clgc.2018.01.016

5. Albiges L, Choueiri T, Escudier B, Galsky M, George D, Hofmann F, et al. A systematic review of sequencing and combinations of systemic therapy in metastatic renal cancer. Eur Urol. (2015) 67:100-10. doi: 10.1016/j.eururo. 2014.04.006

6. Mennitto A, Verzoni E, Grassi P, Ratta R, Fucà G, Procopio G. Multimodal treatment of advanced renal cancer in 2017. Expert Rev Clin Pharmacol. (2017) 10:1395-402. doi: 10.1080/17512433.2017.1386552
The development of biomarkers endowed with high sensitivity, specificity and accuracy able to identify which patients may truly benefit from the treatment with ICIs would allow to refine the therapeutic selection and to better tailor the treatment strategy. Furthermore, recent studies provided evidence that maybe even the prognostic stratification of mRCC patients should be refined in those treated with ICI, including the role of systemic inflammation besides the canonical criteria (74).

The amount of data collected so far in the setting of mRCC, encompassing the expression of PD-L1, the TMB and the widespread role of immunity, has not yet established a predictive biomarker applicable in the clinical practice. The first remark is that probably the huge complexity of the scenario of response to immunotherapy could not be captured by a single biomarker, thus potentially suggesting the need for more comprehensive and integrated approaches. Another point to underline is that the high heterogeneity of the studies conducted, as well as of the techniques and methodologies used and the variety of markers investigated, adds further complexity and limits the validation of these biomarkers with the aim to realize a widely exploitable tool in the clinical setting. Finally, although extremely promising results have been obtained for several biomarkers so far, solid evidence-based data deriving from large, prospective ad hoc clinical trials should be collected in order to change the clinical decision-making process in the everyday real-life.

\section{AUTHOR CONTRIBUTIONS}

AR and GP conceptualized and designed the manuscript. AR drafted the manuscript. PS, EZ, AM, MS, MC, VG, EV, FB, and GP critically reviewed the manuscript. All the authors approved the final version of the manuscript submitted.

7. Topalian SL, Hodi FS, Brahmer JR, Gettinger SN, Smith DC, McDermott DF, et al. Safety, activity, and immune correlates of anti-PD-1 antibody in cancer. N Engl J Med. (2012) 366:2443. doi: 10.1056/NEJMoa1200690

8. Motzer RJ, Escudier B, McDermott DF, George S, Hammers HJ, Srinivas S, et al. Nivolumab versus everolimus in advanced renal-cell carcinoma. $N$ Engl J Med. (2015) 373:1803-13. doi: 10.1056/NEJMoa1510665

9. Escudier B, Sharma P, McDermott DF, George S, Hammers HJ, Srinivas S, et al. CheckMate 025 randomized phase 3 study: outcomes by key baseline factors and prior therapy for nivolumab versus everolimus in advanced renal cell carcinoma. Eur Urol. (2017) 72:962-71. doi: 10.1016/j.eururo.2017.02.010

10. Administration USFD. FDA Expands Use of Immunotherapeutic to Kidney Cancer. (2015). Available online at: http://blog.aacr.org/fda-approvalnivolumab-kidney-cancer/ (accessed November 25, 2015).

11. Agency EM. New Treatment for Advanced Form of Kidney Cancer. (2016). Available online at: https://news.bms.com/press-release/europeancommission-approves-bristol-myers-squibbs-opdivo-nivolumabpreviously-treated (accessed April 6, 2016).

12. Grimm MO, Leucht K, Grünwald V, Foller S. New first line treatment options of clear cell renal cell cancer patients with PD-1 or PD-L1 immune-checkpoint inhibitor-based combination therapies. J Clin Med. (2020) 9:565.

13. Motzer RJ, Tannir NM, McDermott DF, Arén Frontera O, Melichar B, Choueiri TK, et al. Nivolumab plus ipilimumab versus sunitinib in advanced renal-cell carcinoma. N Engl J Med. (2018) 378:1277-90. doi: 10.1056/ NEJMoa1712126 
14. Motzer RJ, Penkov K, Haanen J, Rini B, Albiges L, Campbell MT, et al. Avelumab plus axitinib versus sunitinib for advanced renal-cell carcinoma. $N$ Engl J Med. (2019) 380:1103-15. doi: 10.1056/NEJMoa1816047

15. Rini BI, Powles T, Atkins MB, Escudier B, McDermott DF, Suarez C, et al. Atezolizumab plus bevacizumab versus sunitinib in patients with previously untreated metastatic renal cell carcinoma (IMmotion151): a multicentre, open-label, phase 3, randomised controlled trial. Lancet. (2019) 393:2404-15. doi: 10.1016/S0140-6736(19)30723-8

16. Rini BI, Plimack ER, Stus V, Gafanov R, Hawkins R, Nosov D, et al. Pembrolizumab plus axitinib versus sunitinib for advanced renal-cell carcinoma. N Engl J Med. (2019) 380:1116-27. doi: 10.1056/NEJMoa1816714

17. Administration USFD. FDA Approves Nivolumab Plus Ipilimumab Combination for Intermediate or Poor-Risk Advanced Renal Cell Carcinoma. (2018). Available online at: https://www.fda.gov/drugs/resourcesinformation-approved-drugs/fda-approves-nivolumab-plus-ipilimumabcombination-intermediate-or-poor-risk-advanced-renal-cell (accessed April 16, 2018).

18. Administration USFD. FDA Approves Pembrolizumab Plus Axitinib for Advanced Renal Cell Carcinoma. (2019). Available online at: https://www.fda.gov/drugs/drug-approvals-and-databases/fda-approvespembrolizumab-plus-axitinib-advanced-renal-cell-carcinoma (accessed April 19, 2019).

19. Ravi P, Mantia C, Su C, Sorenson K, Elhag D, Rathi N, et al. Evaluation of the safety and efficacy of immunotherapy rechallenge in patients with renal cell carcinoma. JAMA Oncol. (2020) 20:e202169.

20. Brahmer JR, Drake CG, Wollner I, Powderly JD, Picus J, Sharfman WH, et al. Phase I study of single-agent anti-programmed death-1 (MDX-1106) in refractory solid tumors: safety, clinical activity, pharmacodynamics, and immunologic correlates. J Clin Oncol. (2010) 28:3167-75. doi: 10.1200/JCO. 2009.26.7609

21. Herbst RS, Soria J-C, Kowanetz M, Fine GD, Hamid O, Gordon MS, et al. Predictive correlates of response to the anti-PD-L1 antibody MPDL3280A in cancer patients. Nature. (2014) 515:563-7.

22. Patel SP, Kurzrock R. PD-L1 Expression as a Predictive Biomarker in Cancer Immunotherapy. Mol Cancer Ther. (2015) 14:847-56. doi: 10.1158/1535-7163. MCT-14-0983

23. Wang X, Teng F, Kong L, Yu J. PD-L1 expression in human cancers and its association with clinical outcomes. Onco Targets Ther. (2016) 9:5023-39. doi: 10.2147/OTT.S105862

24. Brody R, Zhang Y, Ballas M, Siddiqui MK, Gupta P, Barker C, et al. PD-L1 Expression in advanced NSCLC: insights into risk stratification and treatment selection from a systematic literature review. Lung Cancer. (2017) 112:200-15. doi: 10.1016/j.lungcan.2017.08.005

25. Thompson RH, Dong H, Kwon ED. Implications of B7-H1 expression in clear cell carcinoma of the kidney for prognostication and therapy. Clin Cancer Res. (2007) 13(2 Pt 2):709-15s. doi: 10.1158/1078-0432.CCR-061868

26. Ueda K, Suekane S, Kurose H, Chikui K, Nakiri M, Nishihara K, et al. Prognostic value of PD-1 and PD-L1 expression in patients with metastatic clear cell renal cell carcinoma. Urol Oncol. (2018) 36:499.e9-e16. doi: 10.1016/ j.urolonc.2018.07.003

27. Kahlmeyer A, Stöhr CG, Hartmann A, Goebell PJ, Wullich B, Wach S, et al. Expression of PD-1 and CTLA-4 are negative prognostic markers in renal cell carcinoma. J Clin Med. (2019) 8:743. doi: 10.3390/jcm8050743

28. Choueiri TK, Figueroa DJ, Fay AP, Signoretti S, Liu Y, Gagnon R, et al. Correlation of PD-L1 tumor expression and treatment outcomes in patients with renal cell carcinoma receiving sunitinib or pazopanib: results from COMPARZ, a randomized controlled trial. Clin Cancer Res. (2015) 21:1071-7. doi: 10.1158/1078-0432.CCR-14- 1993

29. Abdallah F, Xie W, Braun DA, Ficial M, Bakouny Z, Nassar AN, et al. PD-L1 expression and clinical outcomes to cabozantinib, everolimus and sunitinib in patients with metastatic renal cell carcinoma: analysis of the randomized clinical trials METEOR and CABOSUN. Clin Cancer Res. (2019) 25: $6080-8$.

30. Iacovelli R, Nole F, Verri E, Renne G, Paglino C, Santoni M, et al. Prognostic role of $\mathrm{PD}-\mathrm{L} 1$ expression in renal cell carcinoma. a systematic review and meta-analysis. Target Oncol. (2016) 11:143-8. doi: 10.1007/s11523-0150392-7
31. Yarchoan M, Albacker LA, Hopkins AC, Montesion M, Murugesan K, Vithayathil TT, et al. PD-L1 expression and tumor mutational burden are independent biomarkers in most cancers. JCI Insight. (2019) 4:e126908. doi: 10.1172/jci.insight. 126908

32. Zhu J, Armstrong AJ, Friedlander TW, Kim W, Pal SK, George DJ, et al. Biomarkers of immunotherapy in urothelial and renal cell carcinoma: PDL1, tumor mutational burden, and beyond. J Immunother Cancer. (2018) 6:4. doi: 10.1186/s40425-018-0314-1

33. Postow M, Cardona D, Taube J, Anders R, Taylor C, Wolchok J, et al. Peripheral and tumor immune correlates in patients with advanced melanoma treated with nivolumab (anti-PD-1, BMS-936558, ONO-4538) monotherapy or in combination with ipilimumab. J Transl Med. (2014) 12:8. doi: 10.1186/14795876-12-S1-O8

34. Kefford R, Ribas A, Hamid O, Robert C, Daud A, Wolchok JD, et al. Clinical efficacy and correlation with tumor PD-L1 expression in patients (pts) with melanoma (MEL) treated with the anti-PD-1 monoclonal antibody MK-3475. J Clin Oncol. (2014) 32(Suppl. 5):3005.

35. Reck M, Rodríguez-Abreu D, Robinson AG, Hui R, Csõszi T, Fülöp A, et al. Pembrolizumab versus chemotherapy for PD-L1-positive non-smallcell lung cancer. N Engl J Med. (2016) 375:1823-33. doi: 10.1056/NEJMoa160 6774

36. McDermott DF, Huseni MA, Atkins MB, Motzer RJ, Rini BI, Escudier B, et al. Clinical activity and molecular correlates of response to atezolizumab alone or in combination with bevacizumab versus sunitinib in renal cell carcinoma. Nat Med. (2018) 24:749-57. doi: 10.1038/s41591-018-0053-3

37. Choueiri TK, Motzer RJ, Rini BI, Haanen J, Campbell MT, Venugopal B, et al. Updated efficacy results from the JAVELIN Renal 101 trial: first-line avelumab plus axitinib versus sunitinib in patients with advanced renal cell carcinoma. Ann Oncol. (2020) 31:1030-9. doi: 10.1016/j.annonc.2020.04.010

38. Kluger HM, Zito CR, Turcu G, Baine MK, Zhang H, Adeniran A, et al. PD-L1 studies across tumor types, its differential expression and predictive value in patients treated with immune checkpoint inhibitors. Clin Cancer Res. (2017) 23:4270-9. doi: 10.1158/1078-0432.CCR-16-3146

39. Kammerer-Jacquet SF, Deleuze A, Saout J, Mathieu R, Laguerre B, Verhoest G, et al. Targeting the PD-1/PD-L1 pathway in renal cell carcinoma. Int J Mol Sci. (2019) 20:692. doi: 10.3390/ijms20071692

40. Jilaveanu LB, Shuch B, Zito CR, Parisi F, Barr M, Kluger Y, et al. PD-L1 expression in clear cell renal cell carcinoma: an analysis of nephrectomy and sites of metastases. J Cancer. (2014) 5:166-72.

41. Yarchoan M, Hopkins A, Jaffee EM. Tumor mutational burden and response rate to PD-1 inhibition. N Engl J Med. (2017) 377:2500-1. doi: 10.1056/ NEJMc1713444

42. Conway JR, Kofman E, Mo SS, Elmarakeby H, Van Allen E. Genomics of response to immune checkpoint therapies for cancer: implications for precision medicine. Genome Med. (2018) 10:93. doi: 10.1186/s13073-0180605-7

43. Hellmann MD, Ciuleanu T-E, Pluzanski A, Lee JS, Otterson GA, AudigierValette C, et al. Nivolumab plus ipilimumab in lung cancer with a high tumor mutational burden. N Engl J Med. (2018) 378:2093-104. doi: 10.1056/ NEJMoa1801946

44. Snyder A, Makarov V, Merghoub T, Yuan J, Zaretsky JM, Desrichard A, et al. Genetic basis for clinical response to CTLA-4 blockade in melanoma. N Engl J Med. (2014) 371:2189-99. doi: 10.1056/NEJMoa1406498

45. Krieger T, Pearson I, Bell J, Doherty J, Robbins P. Targeted literature review on use of tumor mutational burden status and programmed cell death ligand 1 expression to predict outcomes of checkpoint inhibitor treatment. Diagn Pathol. (2020) 15:6. doi: 10.1186/s13000-020-0927-9

46. de Velasco G, Miao D, Voss MH, Hakimi AA, Hsieh JJ, Tannir NM, et al. Tumor mutational load and immune parameters across metastatic renal cell carcinoma risk groups. Cancer Immunol Res. (2016) 4:820-2. doi: 10.1158/ 2326-6066.CIR-16-0110

47. Alexandrov LB, Nik-Zainal S, Wedge DC, Aparicio SA, Behjati S, Biankin $\mathrm{AV}$, et al. Signatures of mutational processes in human cancer. Nature. (2013) 500:415-21. doi: 10.1038/nature12477

48. Turajlic S, Litchfield K, Xu H, Rosenthal R, McGranahan N, Reading $\mathrm{JL}$, et al. Insertion-and-deletion-derived tumour-specific neoantigens and the immunogenic phenotype: a pan-cancer analysis. Lancet Oncol. (2017) 18:1009-21. doi: 10.1016/S1470-2045(17)30516-8 
49. Zhang C, Li Z, Qi F, Hu X, Luo J. Exploration of the relationships between tumor mutation burden with immune infiltrates in clear cell renal cell carcinoma. Ann Transl Med. (2019) 7:648. doi: 10.21037/atm.2019.10.84

50. Samstein RM, Lee CH, Shoushtari AN, Hellmann MD, Shen R, Janjigian YY, et al. Tumor mutational load predicts survival after immunotherapy across multiple cancer types. Nat Genet. (2019) 51:202-6. doi: 10.1038/s41588-0180312-8

51. Miao D, Margolis CA, Gao W, Liu D, Taylor-Weiner A, Wankowicz SM, et al. Genomic correlates of response to immune checkpoint therapies in clear cell renal cell carcinoma. Science. (2018) 359:801-6. doi: 10.1038/s41588-0180200-2

52. Labriola MK, Zhu J, Gupta R, McCall S, Jackson J, Kong EF, et al. Characterization of tumor mutation burden, PD-L1 and DNA repair genes to assess relationship to immune checkpoint inhibitors response in metastatic renal cell carcinoma. J Immunother Cancer. (2020) 8:e000319. doi: 10.1136/ jitc-2019-000319

53. Masini C, Iotti C, De Giorgi U, Bellia RS, Buti S, Salaroli F, et al. Nivolumab (NIVO) in combination with stereotactic body radiotherapy (SBRT) in pretreated patients (pts) with metastatic renal cell carcinoma (mRCC): First results of phase II NIVES study. J Clin Oncol. (2020) 38:613-613.

54. Zhu Q, Cai MY, Weng DS, Zhao JJ, Pan QZ, Wang QJ, et al. PD-L1 expression patterns in tumour cells and their association with CD8+ tumour infiltrating lymphocytes in clear cell renal cell carcinoma. J Cancer. (2019) 10:1154-61. doi: $10.7150 /$ jca. 29052

55. Kawashima A, Uemura M, Nonomura N. Importance of multiparametric evaluation of immune-related t-cell markers in renal-cell carcinoma. Clin Genitourin Cancer. (2019) 17:e1147-52. doi: 10.1016/j.clgc.2019.07.021

56. Zhang S, Zhang E, Long J, Hu Z, Peng J, Liu L, et al. Immune infiltration in renal cell carcinoma. Cancer Sci. (2019) 110:1564-72. doi: 10.1111/cas.13996

57. Şenbabaoğlu Y, Gejman RS, Winer AG, Liu M, Van Allen EM, de Velasco $\mathrm{G}$, et al. Tumor immune microenvironment characterization in clear cell renal cell carcinoma identifies prognostic and immunotherapeutically relevant messenger RNA signatures. Genome Biol. (2016) 17:231. doi: 10.1186/s13059016-1092-z

58. Yao J, Xi W, Zhu Y, Wang H, Hu X, Guo J. Checkpoint molecule PD1-assisted CD8+ T lymphocyte count in tumor microenvironment predicts overall survival of patients with metastatic renal cell carcinoma treated with tyrosine kinase inhibitors. Cancer Manag Res. (2018) 10:3419-3431. doi: 10. 2147/CMAR.S172039

59. Nakano O, Sato M, Naito Y, Suzuki K, Orikasa S, Aizawa M, et al. Proliferative activity of intratumoral CD8(+) T-lymphocytes as a prognostic factor in human renal cell carcinoma: clinicopathologic demonstration of antitumor immunity. Cancer Res. (2001) 61:5132-6.

60. Giraldo NA, Becht E, Pagès F, Skliris G, Verkarre V, Vano Y, et al. Orchestration and prognostic significance of immune checkpoints in the microenvironment of primary and metastatic renal cell cancer. Clin Cancer Res. (2015) 21:303140. doi: 10.1158/1078-0432.CCR-14-2926

61. Hamada T, Soong TR, Masugi Y, Kosumi K, Nowak JA, da Silva A, et al. TIME (Tumor Immunity in the MicroEnvironment) classification based on tumor CD274 (PD-L1) expression status and tumorinfiltrating lymphocytes in colorectal carcinomas. Oncoimmunology. (2018) 7:e1442999. doi: 10.1080/ 2162402X.2018.1442999

62. Zhu G, Pei L, Yin H, Lin F, Li X, Zhu X, et al. Profiles of tumor-infiltrating immune cells in renal cell carcinoma and their clinical implications. Oncol Lett. (2019) 18:5235-42. doi: 10.3892/ol.2019.10896

63. Cristescu R, Mogg R, Ayers M, Albright A, Murphy E, Yearley J, et al. Pan-tumor genomic biomarkers for PD-1 checkpoint blockade-based immunotherapy. Science. (2018) 362:eaar3593. doi: 10.1126/science.aar3593

64. Ascierto ML, McMiller TL, Berger AE, Danilova L, Anders RA, Netto GJ, et al. The intratumoral balance between metabolic and immunologic gene expression is associated with anti-PD-1 response in patients with renal cell carcinoma. Cancer Immunol Res. (2016) 4:726-33. doi: 10.1158/2326-6066. CIR-16-0072

65. Wallin JJ, Bendell JC, Funke R, Sznol M, Korski K, Jones S, et al. Atezolizumab in combination with bevacizumab enhances antigen-specific T-cell migration in metastatic renal cell carcinoma. Nat Commun. (2016) 7:12624. doi: 10.1038/ ncomms 12624

66. Abdin SM, Zaher DM, Arafa EA, Omar HA. Tackling cancer resistance by immunotherapy: updated clinical impact and safety of PD-1/PD-L1 Inhibitors. Cancers (Basel). (2018) 10:32. doi: 10.3390/cancers10020032

67. Brahmer JR, Lacchetti C, Schneider BJ, Atkins MB, Brassil KJ, Caterino JM, et al. Management of immune-related adverse events in patients treated with immune checkpoint inhibitor therapy: american society of clinical oncology clinical practice guideline. J Clin Oncol. (2018) 36:1714-68. doi: 10.1200/JCO. 2017.77.6385

68. Michot JM, Bigenwald C, Champiat S, Collins M, Carbonnel F, Postel-Vinay $\mathrm{S}$, et al. Immune-related adverse events with immune checkpoint blockade: a comprehensive review. Eur J Cancer. (2016) 54:139-48.

69. Wang PF, Chen Y, Song SY, Wang TJ, Ji WJ, Li SW, et al. Immune-related adverse events associated with anti-pd-1/pd-11 treatment for malignancies: a meta-analysis. Front Pharmacol. (2017) 8:730. doi: 10.3389/fphar.2017. 00730

70. Downey SG, Klapper JA, Smith FO, Yang JC, Sherry RM, Royal RE, et al. Prognostic factors related to clinical response in patients with metastatic melanoma treated by CTL-associated antigen-4 blockade. Clin Cancer Res. (2007) 13(22 Pt 1):6681-8. doi: 10.1158/1078-0432.CCR-07-0187

71. Teraoka S, Fujimoto D, Morimoto T, Kawachi H, Ito M, Sato Y, et al. Early immune-related adverse events and association with outcome in advanced non-small cell lung cancer patients treated with nivolumab: a prospective cohort study. J Thorac Oncol. (2017) 12:1798-805. doi: 10.1016/j.jtho.2017. 08.022

72. Verzoni E, Carteni G, Cortesi E, Giannarelli D, De Giglio A, Sabbatini R, et al. Real-world efficacy and safety of nivolumab in previously-treated metastatic renal cell carcinoma, and association between immune-related adverse events and survival: the Italian expanded access program. J Immunother Cancer. (2019) 7:99. doi: 10.1186/s40425-019-0579-Z

73. Ishihara H, Takagi T, Kondo T, Homma C, Tachibana H, Fukuda H, et al. Association between immune-related adverse events and prognosis in patients with metastatic renal cell carcinoma treated with nivolumab. Urol Oncol. (2019) 37:355.e21-e29. doi: 10.1016/j.urolonc.2019.03.003

74. Martini DJ, Liu Y, Shabto JM, Carthon BC, Hitron EE, Russler GA, et al. Novel risk scoring system for patients with metastatic renal cell carcinoma treated with immune checkpoint inhibitors. Oncologist. (2020) 25:e484-91.

Conflict of Interest: GP declares receiving honoraria for advisory board from Bayer, Bristol Myers Squibb, Ipsen, Merck, Novartis, and Pfizer. EV declares receiving honoraria for advisory board from Pfizer, Bristol Myers Squibb, Ipsen, and EUSA pharm. FB reported receiving honoraria for speaker activities and participation in advisory boards from Amgen, Inc., Roche, and Novartis International AG.

The remaining authors declare that the research was conducted in the absence of any commercial or financial relationships that could be construed as a potential conflict of interest.

Copyright (C) 2020 Raimondi, Sepe, Zattarin, Mennitto, Stellato, Claps, Guadalupi, Verzoni, de Braud and Procopio. This is an open-access article distributed under the terms of the Creative Commons Attribution License (CC BY). The use, distribution or reproduction in other forums is permitted, provided the original author(s) and the copyright owner(s) are credited and that the original publication in this journal is cited, in accordance with accepted academic practice. No use, distribution or reproduction is permitted which does not comply with these terms. 